\title{
Accelerating AdS black holes as the holographic heat engines in a benchmarking scheme
}

\author{
Jialin Zhang, Yanjun Li, Hongwei Yu ${ }^{\mathrm{a}}$ \\ Department of Physics and Synergetic Innovation Center for Quantum Effects and Applications, Hunan Normal University, Changsha 410081, \\ Hunan, China
}

Received: 18 April 2018 / Accepted: 4 August 2018 / Published online: 13 August 2018

(C) The Author(s) 2018

\begin{abstract}
We investigate the properties of holographic heat engines with an uncharged accelerating non-rotating AdS black hole as the working substance in a benchmarking scheme. We find that the efficiencies of the black hole heat engines can be influenced by both the size of the benchmark circular cycle and the cosmic string tension as a thermodynamic variable. In general, the efficiency can be increased by enlarging the cycle, but is still constrained by a universal bound $2 \pi /(\pi+4)$ as expected. A cross-comparison of the efficiencies of the accelerating black hole heat engines and Schwarzschild-AdS black hole heat engines suggests that the acceleration also increases the efficiency although the amount of increase is not remarkable.
\end{abstract}

\section{Introduction}

Black holes are fascinating objects which provide a useful link to explore the relationship between general relativity, thermodynamics and quantum theory. More than four decades after Hawking's discovery of black hole radiation [1,2], the black hole thermodynamics has been established and developed into an important sub-discipline in physics. The study of black hole thermodynamics has already shed some light on the nature of quantum gravity in the lack of a consistent quantum theory of it. Recently, it is found that the thermodynamical properties of black holes in Antide Sitter (AdS) space are quite different from those in flat or de Sitter(dS) space, which are thermodynamically stable, on one hand, and on the other hand, deep insight has been gained into some phenomena in strongly coupled quantum field theories by means of AdS/CFT correspondence [3-8].

So far, many approaches have been introduced to analyze black hole thermodynamics, such as, those of positing the laws of gravitation to be connected with the laws of

\footnotetext{
a e-mail: hwyu@hunnu.edu.cn
}

thermodynamics $[9,10]$, treating the black holes as a holographically dual system in quantum chromodynamics [11] and condensed matter physics $[12,13]$, and approaching the thermodynamics of black holes geometrically [14-23]. More recently, by elevating the negative cosmological constant $\Lambda$ as the pressure and defining the thermodynamic volume satisfying a reverse isoperimetric inequality $[24,25]$ as the conjugate to the pressure in the extended black hole thermodynamics [24,26-30], some interesting thermodynamic phenomena and rich phase structures quite analogous to the van der Waals fluids are discovered [31], and this burgeoning subject, which named as black hole chemistry $[32,33]$, has attracted a lot of attention.

In the context of black hole chemistry, Johnson proposed the concept of holographic heat engines which can extract work with AdS black holes used as the work materials in the pressure-volume phase space [34]. The name of "holographic" originates from the fact that the cycle represents a journey defined on the space of dual field theories in one dimension lower [34]. After Johnson's pioneering work, subsequent studies have generalized this concept to other black holes [35-48]. More recently, in order to better compare the efficiency of the heat engines with different black holes as working substances, Chakraborty and Johnson introduced a circular cycle of the heat engine in the $P-V$ phase space to benchmark black hole heat engines [49]. Since the circular cycle of a heat engine is a judicious choice for all working substances without favoring any species of black holes, it can be considered as a benchmarking cycle in general.

In this paper, we plan to generalize, in the benchmarking scheme, the study of holographic heat engines to the case of AdS black holes with acceleration. The accelerating black holes are known to be described by the so-called $C$-metric [50-53], which has been used to investigate the pair creation of black holes [54], the splitting of cosmic strings $[55,56]$, and even to construct the black ring in five- 
dimensional gravity [57]. Recently, Appels et al. have derived the thermodynamics of accelerating black holes [58] and generalized the results to the case of varying conical deficits for $C$-metric $[59,60]$.

The paper is organized as follows. We will review the thermodynamics of accelerating black holes with conical defects in $C$-metric in next section. In Sect. 3, we will study the benchmarking holographic heat engines with accelerated AdS black holes as working substances with numerical analysis. We will summarize and conclude in Sect. 4 .

\section{A slowly accelerating AdS black hole and its thermodynamics}

Let us now give a brief review of an uncharged slowly accelerating AdS black hole and its thermodynamics. The accelerated AdS black holes can be described by $C$-metric $[58,59]$. However, in order to have a well-defined temperature for these black holes, it is appropriated to restrict the acceleration to be slow (slowly accelerating $C$-metric) so that the acceleration horizon can be negated by a negative cosmological constant and only the black hole horizon exists [59].

Then, a slowly accelerating AdS black hole can be described by the following $C$-metric $[53,58,59]$

$d s^{2}=\frac{1}{\Omega^{2}}\left[f(r) d t^{2}-\frac{d r^{2}}{f(r)}-r^{2}\left(\frac{d \theta^{2}}{g(\theta)}+g(\theta) \sin ^{2} \theta \frac{d \phi^{2}}{K^{2}}\right)\right]$,

where

$f(r)=\left(1-A^{2} r^{2}\right)\left(1-\frac{2 m}{r}\right)+\frac{r^{2}}{\ell^{2}}$,

$g(\theta)=1+2 m A \cos \theta$,

and the conformal factor $\Omega=1+A r \cos \theta$. Here, the parameters $m$ and $A$ are related to the mass and the magnitude of acceleration of the black hole respectively, $K$ characterizes the conical deficit of the spacetime, and $\ell$ represents the AdS radius. For the slowly accelerating case, technically , if $A \ell<3 \sqrt{3} / 4 \sqrt{2}$, there is only the black hole event horizon $r_{+}$which satisfies $f\left(r_{+}\right)=0$ [59]. It is easy to see that there is a conical deficit in this spacetime which is unequal at two different poles, and it is this difference of the deficits that produces an overall force that drives the acceleration. In fact, if we require that the angular part of the metric be regular at a pole, then we have

$K_{ \pm}=1 \pm 2 m A$

which indicates clearly that we can not have regularity at both poles if $2 m A \neq 0$, and this kind of irregularity along an axis is precisely a definition of a conical singularity that signals the existence of a cosmic string. Actually, the tension of the string $\mu$ is related to the conical deficit angle $\delta$ by $\mu=\delta / 8 \pi$, where

$\delta=2 \pi\left[1-\frac{g(\theta)}{K}\right]$.

So, the string tension varies as we move along the axis, and on the north pole $\left(\theta_{+}=0\right)$ and the south pole $\left(\theta_{-}=\pi\right)$, the cosmic string tensions are given by

$\mu_{ \pm}=\frac{1}{4}-\frac{g\left(\theta_{ \pm}\right)}{4 K}=\frac{1}{4}-\frac{1 \pm 2 m A}{4 K}$.

In order to avoid the occurrence of negative tension defects, the requirement of $\mu_{+} \geq 0$ is compulsory. Clearly, it is easy to find out that $\mu_{+} \leq \mu_{-} \leq 1 / 4$.

In order to obtain the correct thermodynamics, it has been argued that the normalization of the timelike Killing vector should be appropriately chosen [61]. In fact, such a normalization of the time coordinate can be obtained such that it corresponds to the "time" of an asymptotic observer, $\tau=\alpha t$, with $\alpha=\sqrt{1-A^{2} \ell^{2}}$ [60]. The black hole mass associated with the normalized time can be found by using the method of conformal completion $[62,63]$,

$M=\alpha \frac{m}{K}$.

It should be pointed out that $M$ is usually identified with enthalpy rather than internal energy in extended black hole thermodynamics. As usual, the temperature $T$ is given by using the conventional Euclidean method associated with the normalized time $\tau$,

$T=\frac{f^{\prime}\left(r_{+}\right)}{4 \alpha \pi}=\frac{m}{2 \pi \alpha r_{+}^{2}}+\frac{A^{2} m}{2 \alpha \pi}-\frac{A^{2} r_{+}}{2 \alpha \pi}+\frac{r_{+}}{2 \pi \alpha \ell^{2}}$.

And the entropy $S$ of the accelerating black hole still obeys the area theorem

$S=\frac{\pi r_{+}^{2}}{K\left(1-A^{2} r_{+}^{2}\right)}$.

The thermodynamic pressure associated with the cosmological constant in extended black hole thermodynamics reads

$P=-\frac{\Lambda}{8 \pi}=\frac{3}{8 \pi \ell^{2}}$,

and the thermodynamic volume is

$V=\frac{4 \pi}{3 K \alpha}\left[\frac{r_{+}^{3}}{\left(1-A^{2} r_{+}^{2}\right)^{2}}+m A^{2} \ell^{4}\right]$. 
Then, if we allow the tension of the string to vary, the first law can be straightforwardly derived [60]

$\delta M=T \delta S+V \delta P-\lambda_{+} \delta \mu_{+}-\lambda_{-} \delta \mu_{-}$,

where

$\lambda_{ \pm}=\frac{1}{\alpha}\left[\frac{r_{+}}{1-A^{2} r_{+}^{2}}-m\left(1 \pm \frac{2 A \ell^{2}}{r_{+}}\right)\right]$.

Here, $\lambda_{ \pm}$is defined as a thermodynamic length [59,60], which is conjugate to the tension $\mu_{ \pm}$.

As we have seen, all the thermodynamic variables are the certain combinations of the solution parameters. A change of solution parameter $A$ may lead to changes of the thermodynamic mass $M$ and the cosmic string tensions, so does the change of other solution parameters. Due to the complicated combinations of the thermodynamic variables, it is quite a challenge to obtain the analytic expression for the efficiency of benchmarking black hole heat engines.

\section{Benchmarking black hole heat engines and efficiency}

For a valid cycle of a holographic heat engine, the efficiency is defined by

$\eta=1-\frac{Q_{C}}{Q_{H}}$,

where $Q_{C}$ denotes a net output heat flow in one cycle, and $Q_{H}$ represents a net input heat flow. In general, we can compute the efficiency by keeping the corresponding tensions fixed through the heat engine cycle, then the enthalpy $M$ can be considered as a function of $S$ and $P$.

In the benchmarking scheme of black hole heat engines $[38,48]$, a circular or elliptical cycle has been suggested in order to allow for cross-comparison of the efficiencies of holographic heat engines with different black holes as working substances. The cycle is described by the following parameterized equation in the $P-V$ plane,

$P(\theta)=P_{0}(1+p \sin \theta), \quad V(\theta)=V_{0}(1+v \cos \theta)$,

where $\left(V_{0}, P_{0}\right)$ is the center of the cycle. Strictly speaking, the precise shape of the closed contour in the $P-V$ plane should be elliptical since the units of $P$ and $V$ are different from each other.

For the special case of $C_{V}=0$, i.e., the specific heat capacity at constant volume is vanishing, then enthalpy $M$ can be considered as a function of $V$ and $P$. By tilling the circular/elliptical cycle with a series of rectangles, a net output heat flow can be written in a simple form by applying Eq. (15)

$$
\begin{aligned}
Q_{C}= & M\left(V_{0}(1+v), P_{0}\right)-M\left(V_{0}(1-v), P_{0}\right) \\
& -\frac{\pi P_{0} V_{0} p v}{2} .
\end{aligned}
$$

Performing a similar algorithmic manipulation, we can also get $Q_{H}$. Then the efficiency of the benchmarking black hole heat engine can be calculated exactly. However, for rotating black holes or the case with multiple complicated thermodynamic variables, it is not easy to exactly determine which part of the cycle curve is related to the output heat flow, then Eq. (16) may be inapplicable to determining the net output heat flow, and a numerical integration of $T d S$ will be needed [48].

For convenience, we can adjust the unit of each coordinate axis in the $P-V$ plane so that the shape of the cycle contour looks like a circle with a radius of a numerical value $R$. Then the circular cycle Eq. (15) reads

$P(\theta)=P_{0}+R \sin \theta, \quad V(\theta)=V_{0}+R \cos \theta$.

In order to keep the pressure and volume positive on the circular cycle, the radius $R$ needs to be constrained by the circle center $P_{0}$ and $V_{0}$, i.e., $R<P_{0}$ and $R<V_{0}$. In the following, we will examine properties of heat engines of an accelerated AdS black hole by numerical estimations.

Now, without loss of generality, we choose $K=K_{+}=$ $g\left(\theta_{+}\right)=1+2 m A$ in Eq. (6) so that the metric is regular on the north pole (i.e., $\mu_{+}=0$ ), then the cosmic string tension on the south pole becomes

$\mu_{-}=\frac{K-1}{2 K}$.

For fixed tension, the corresponding cycle contour in the $T-S$ plane can also be directly obtained by some algebraic manipulations of the thermodynamic variables (see Fig. 1).

According to Eqs. (9) and (11), it is not difficult to deduce that $C_{V} \neq 0$ in the case of accelerated black holes. Therefore, the numerical treatment should be called for.

In Fig. 2, we display the relation between the efficiencies of holographic heat engines and the size of the circular cycle in the $P-V$ plane. Here, the Carnot efficiency is defined by $\eta_{C}=1-T_{C} / T_{H}$ with maximum temperature $T_{H}$ and minim temperature $T_{C}$ in the entire cycle process. We can see from the figure that both the efficiency $\eta$ and Carnot efficiency $\eta_{C}$ grow with the increasing radius $R$ and the Carnot efficiency is always higher than the efficiency of benchmarking black hole heat engines. Furthermore, the larger the radius of the circle, the bigger the difference. Therefore, the efficiency $\eta$ cannot approach the Carnot efficiency $\eta_{C}$ even when the working area of the $P-V$ plane becomes larger. Besides these, it is worth noting that the universal upper-bound proposed in Ref. [48] is still valid, i.e., 


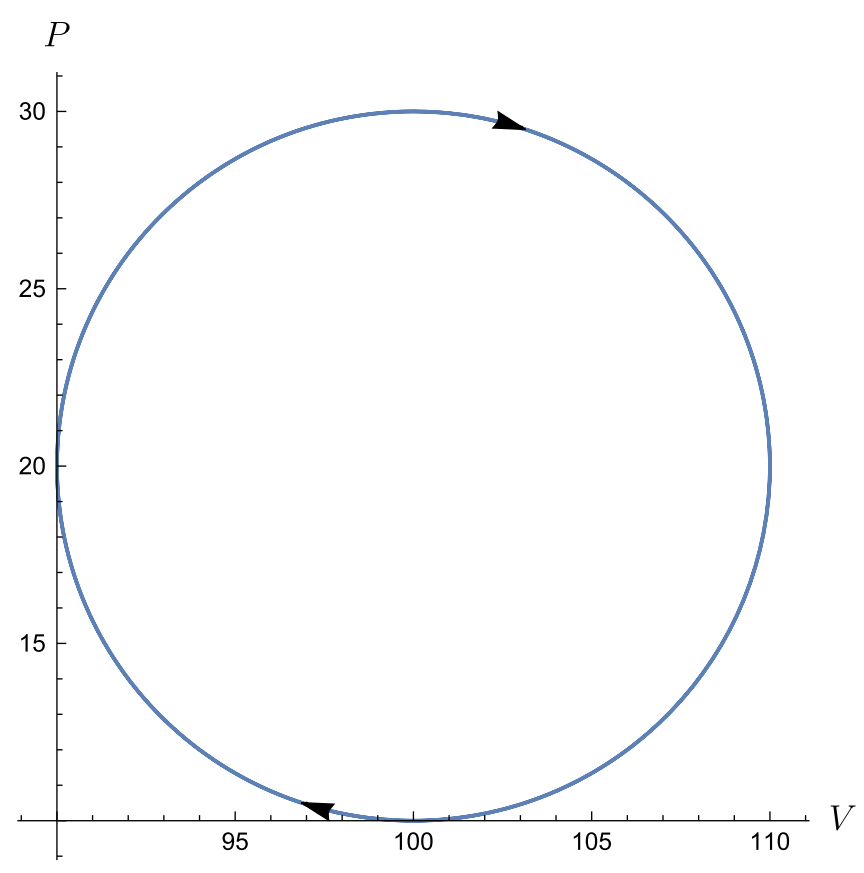

(a)

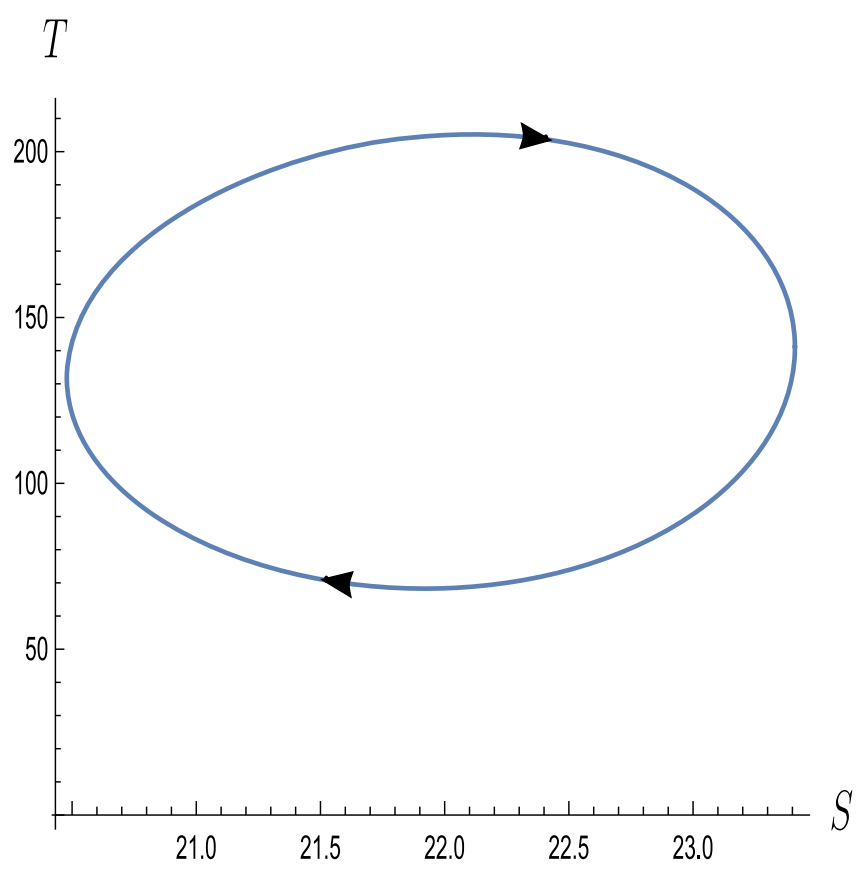

(b)

Fig. 1 For fixed tension $\mu_{-}=0.2$, the corresponding cycle diagram of an accelerating black hole heat engine is respectively plotted in a $P-V$ plane and $\mathbf{b} T-S$ plane. Here, the center of the circle in $P-V$ plane is $\left(V_{0}, P_{0}\right)=(100,20)$ with the radius $R=10$

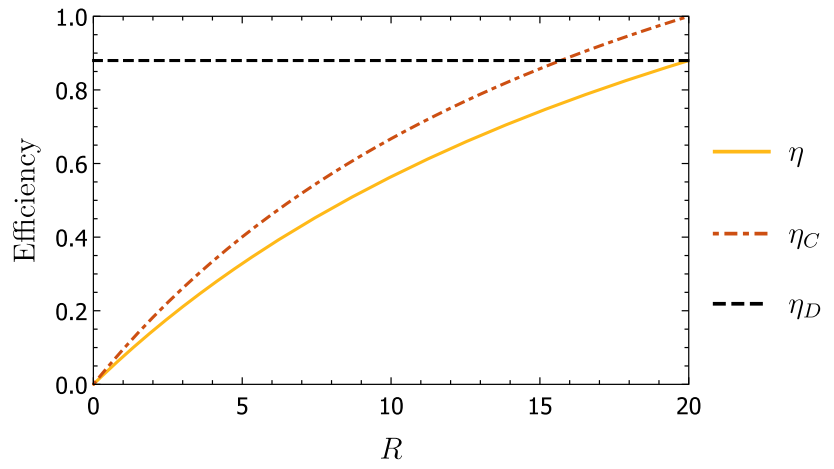

Fig. 2 The efficiency of a heat engine of accelerating black holes is plotted as a function of radius $R$. Here, we assume the center of the circular cycle in $P-V$ plane is $\left(V_{0}, P_{0}\right)=(100,20)$ with the cosmic string tension $\mu_{-}=0.2$, then the radius for a valid heat engine cycle should satisfy $R<20$. Note that $\eta_{C}$ denotes Carnot efficiency and $\eta_{D}$ means the upper-bound value of efficiency

$\eta \leq \eta_{D}=\frac{2 \pi}{\pi+4}$,

and the efficiency $\eta$ approaches the upper-bound in the limit of $R \rightarrow P_{0}$.

For a fixed circular cycle radius, we have plotted the benchmarking heat engine efficiency as a function of the cosmic string tension in Fig. 3, which shows that a stronger cosmic string tension will in principle lead to a somewhat higher efficiency, but the change in efficiency is actually insignifi-

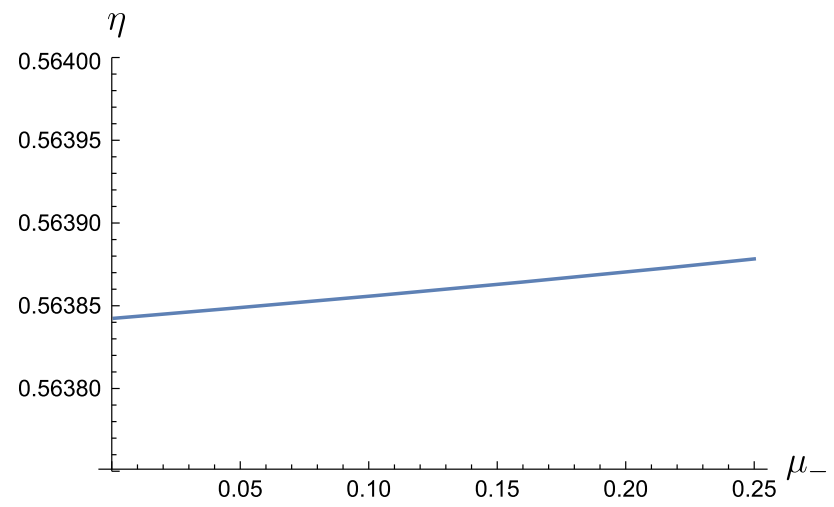

Fig. 3 The efficiency of a heat engine of accelerating black holes is plotted as a function of $\mu_{-}$. Here, the circular cycle is assumed to satisfy $R=10$ with origin at $\left(V_{0}, P_{0}\right)=(100,20)$

cant since the difference shows up only in the 5th significant figure.

Now, we cross-compare the efficiency of different black hole heat engines in the benchmarking scheme. The black hole heat engines we choose are those of the slowly accelerating AdS black holes we just studied, the ideal gas black holes and the Schwarzschild-AdS black holes. We plot the efficiency of the benchmarking heat engine as a function of the radius $R$ with different black holes as working substances in Fig. 4. It is worth noting that both the ideal gas black hole and Schwarzschild-AdS black hole have a vanishing specific heat at constant volume (i.e., $C_{V}=0$, the detailed thermody- 


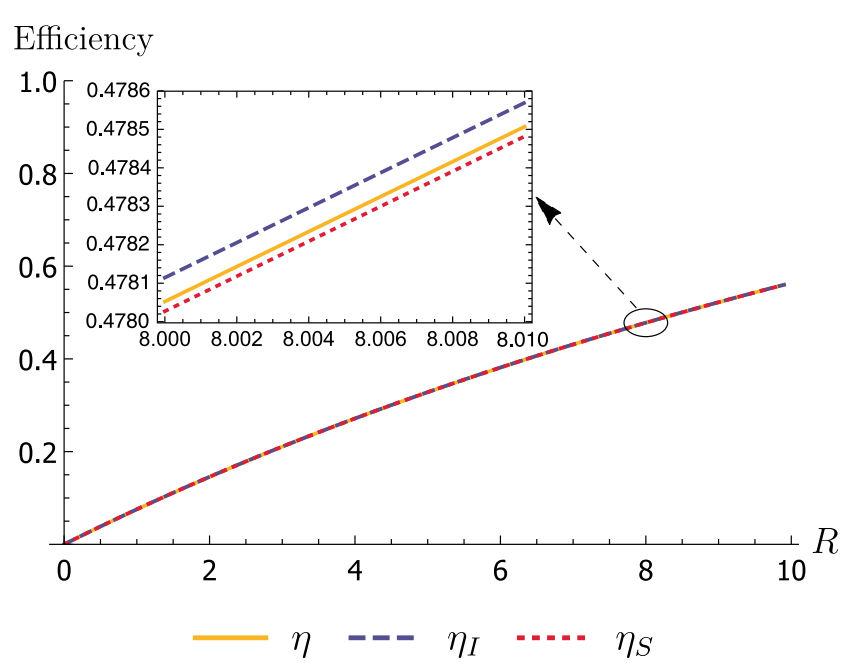

(a)

Fig. 4 a The efficiencies vs the radius $R$ for three different families of black holes as the working material, including accelerating AdS (denoted by $\eta$ with $\mu_{-}=0.2$ ), the ideal gas (denoted by $\eta_{I}$ ) and Schwarzschild-AdS (denoted by $\eta_{S}$ ) black holes. The differences in the

namic quantities can be found in Refs. [31,48,49]), and the holographic heat engine efficiency, which can be obtained directly by using Eq. (16) in the benchmarking scheme, has been studied in Ref. [48].

Figure 4 reveals that the work efficiency of holographic heat engines of slowly accelerating black holes, in general, is larger than that of Schwarzschild-AdS black holes, but smaller than that of ideal gas black holes. Since in the zero acceleration limit the metric (1) approaches that of a Schwarzschild-AdS black hole, one may conclude that with acceleration, the holographic heat engine efficiency is usually larger than that without although the difference is tiny.

\section{Conclusion}

We have explored the properties of holographic heat engines with accelerating black holes as the working substances. This family of accelerating AdS black holes described by $C$ metric represents a black hole with conical deficits along one axis. These conical deficits provide a driving force to generate the acceleration of a black hole. Physically, the topological defect originated from the conical deficit can be interpreted as a finite-width cosmic string core. Due to the fact that all thermodynamic variables of the accelerating black holes are non-linear combinations of the solution parameters $r_{+}, m, \ell$ and $A$, it is quite a challenge to obtain an analytical expression for the efficiency of the holographic heat engines and numerical estimations are resorted to.

In a benchmarking scheme, we have examined the influence of the size of a benchmarking circular cycle and the cos-

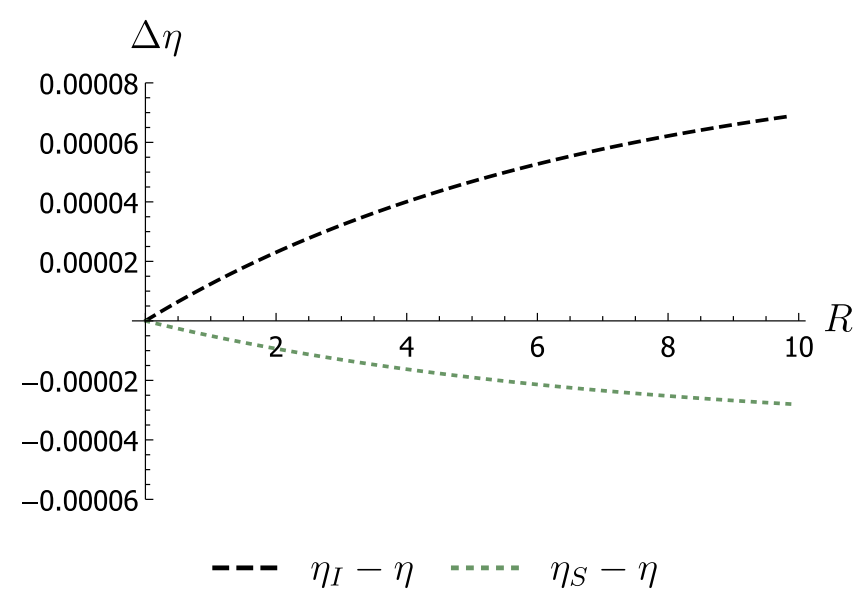

(b)

efficiencies are described in $\mathbf{b}$, where $\eta_{I}-\eta$ and $\eta_{S}-\eta$ are represented by the black dashed line and the green dotted line respectively. Note that all the benchmarking circular cycle is centered at $\left(V_{0}, P_{0}\right)=(100,20)$

mic string tension on the efficiency of black hole heat engines. We find that the efficiency can be increased by enlarging the cycle, but the efficiency cannot exceed the Carnot efficiency as we would expect. Moreover, it is also constrained by a universal bound $2 \pi /(\pi+4)$ proposed in Ref. [48]. When the cosmic string tension is varied, the efficiency in principle increases with the increasing cosmic string tension, but the amount of increase is tiny.

A cross-comparison of the holographic heat engines with slowly accelerating AdS black holes and the SchwarzschildAdS black holes in a same benchmarking cycle shows that the presence of acceleration also increases the efficiency somewhat. Finally, it is worth pointing out that the thermodynamics and the behavior of holographic heat engines, when the accelerating black hole is charged, is an interesting but nontrivial issue, which we would rather leave to a future work.

Acknowledgements This work was supported by the National Natural Science Foundation of China under Grants nos. 11435006 and 11690034.

Open Access This article is distributed under the terms of the Creative Commons Attribution 4.0 International License (http://creativecomm ons.org/licenses/by/4.0/), which permits unrestricted use, distribution, and reproduction in any medium, provided you give appropriate credit to the original author(s) and the source, provide a link to the Creative Commons license, and indicate if changes were made. Funded by SCOAP ${ }^{3}$.

\section{References}

1. S. Hawking, Commun. Math. Phys. 43, 199-220 (1975)

2. S. Hawking, Phys. Rev. D 13, 191-197 (1976) 
3. J.M. Maldacena, Adv. Theor. Math. Phys. 2, 231 (1998)

4. J.M. Maldacena, Int. J. Theor. Phys. 38, 1113 (1999)

5. S.S. Gubser, I.R. Klebanov, A.M. Polyakov, Phys. Lett. B 428, 105 (1998)

6. E. Witten, Adv. Theor. Math. Phys. 2, 253 (1998)

7. E. Witten, Adv. Theor. Math. Phys. 2, 505 (1998)

8. O. Aharony, S.S. Guber, J. Maldacena, H. Ooguri, Y. Oz, Phys. Rep. 323, 183 (2000)

9. T. Jacobson, Phys. Rev. Lett. 75, 1260 (1995)

10. T. Padmanabhan, Rep. Prog. Phys. 73, 046901 (2010)

11. P.K. Kovtun, D.T. Son, A.O. Starinets, Phys. Rev. Lett. 94, 111601 (2005)

12. S.A. Hartnoll, P.K. Kovtun, M. Muller, S. Sachdev, Phys. Rev. B 76, 144502 (2007)

13. S.A. Hartnoll, C.P. Herzog, G.T. Horowitz, Phys. Rev. Lett. 101, 031601 (2008)

14. J.E. Aman, I. Bengtsson, N. Pidokrajt, Gen. Relativ. Gravit. 35, 1733 (2003)

15. H. Quevedo, Gen. Relativ. Gravit. 40, 971 (2008)

16. G. Ruppeiner, Springer Proc. Phys. 153, 179 (2014)

17. S.A.H. Mansoori, B. Mirza, Eur. Phys. J. C 74, 2681 (2014)

18. J. Suresh, R. Tharanath, N. Varghese, V.C. Kuriakose, Eur. Phys. J. C 74, 2819 (2014)

19. J.-L. Zhang, R.-G. Cai, H. Yu, JHEP 02, 143 (2015)

20. J.-L. Zhang, R.-G. Cai, H. Yu, Phys. Rev. D 91, 044028 (2015)

21. S.H. Hendi, A. Sheykhi, S. Panahiyan, B.E. Panah, Phys. Rev. D 92, 064028 (2015)

22. C. Gruber, O. Luongo, H. Quevedo, arXiv:1603.09443 [gr-qc] (2016)

23. R. Banerjee, B.R. Majhi, S. Samanta, Phys. Lett. B 767, 25 (2017)

24. M. Cvetic, G.W. Gibbons, D. Kubiznak, C.N. Pope, Phys. Rev. D 84, 024037 (2011)

25. R.A. Hennigar, R.B. Mann, D. Kubiznak, Phys. Rev. Lett. 115, 031101 (2015)

26. C. Teitelboim, Phys. Lett. B 158, 293 (1985)

27. M.M. Caldarelli, G. Cognola, D. Klemm, Class. Quantum Gravity 17, $399(2000)$

28. D. Kastor, S. Ray, J. Traschen, Class. Quantum Gravity 26, 195011 (2009)

29. B.P. Dolan, Class. Quantum Gravity 28, 125020 (2011)

30. B.P. Dolan, Class. Quantum Gravity 28, 235017 (2011)

31. D. Kubiznak, R.B. Mann, M. Teo, Class. Quantum Gravity 34, 063001 (2017)
32. D. Kubiznak, R.B. Mann, Can. J. Phys. 93, 999 (2015)

33. R.B. Mann, Springer Proc. Phys. 170, 197 (2016)

34. C.V. Johnson, Class. Quantum Gravity 31, 205002 (2014)

35. A. Belhaj, M. Chabab, H. El Moumni, K. Masmar, M.B. Sedra, A. Segui, JHEP 05, 149 (2015)

36. E. Caceres, P.H. Nguyen, J.F. Pedraza, JHEP 09, 184 (2015)

37. M.R. Setare, H. Adami, Gen. Relativ. Gravit. 47, 133 (2015)

38. C.V. Johnson, Class. Quantum Gravity 33, 215009 (2016)

39. C.V. Johnson, Class. Quantum Gravity 33, 135001 (2016)

40. M. Zhang, W.-B. Liu, Int. J. Theor. Phys. 55, 5136 (2016)

41. J. Sadeghi, K. Jafarzade, Int. J. Theor. Phys. 56, 3387 (2017)

42. S.-W. Wei, Y.-X. Liu, arXiv:1708.08176 [gr-qc] (2017)

43. S. H. Hendi, B. Eslam Panah, S. Panahiyan, H. Liu, X. H. Meng, Phys. Lett. B 781, 40 (2018)

44. C. Bhamidipati, P.K. Yerra, Phys. Lett. B 772, 800 (2017)

45. H. Xu, Y. Sun, L. Zhao, Int. J. Mod. Phys. D 26, 1750151 (2017)

46. H. Liu, X.-H. Meng, Eur. Phys. J. C 77, 556 (2017)

47. J.-X. Mo, F. Liang, G.-Q. Li, JHEP 03, 010 (2017)

48. R.A. Hennigar, F. McCarthy, A. Ballon, R.B. Mann, Class. Quantum Gravity 34, 175005 (2017)

49. A. Chakraborty, C. V. Johnson, arXiv:1612.09272 [hep-th] (2016)

50. W. Kinnersley, M. Walker, Phys. Rev. D 2, 1359 (1970)

51. J.F. Plebanski, M. Demianski, Ann. Phys. (NY) 98, 98 (1976)

52. O.J.C. Dias, J.P.S. Lemos, Phys. Rev. D 67, 064001 (2003)

53. J.B. Griffiths, J. Podolsky, Int. J. Mod. Phys. D 15, 335 (2006)

54. F. Dowker, J.P. Gauntlett, D.A. Kastor, J.H. Traschen, Phys. Rev. D 49, 2909 (1994)

55. R. Gregory, M. Hindmarsh, Phys. Rev. D 52, 5598 (1995)

56. D.M. Eardley, G.T. Horowitz, D.A. Kastor, J.H. Traschen, Phys. Rev. Lett. 75, 3390 (1995)

57. R. Emparan, H.S. Reall, Phys. Rev. Lett. 88, 101101 (2002)

58. M. Appels, R. Gregory, D. Kubiznak, Phys. Rev. Lett. 117, 131303 (2016)

59. M. Appels, R. Gregory, D. Kubiznak, JHEP 05, 116 (2017)

60. A. Anabalon, M. Appels, R. Gregory, D. Kubiznak, R.B. Mann, A. Ovgun, arXiv:1805.02687 [hep-th] (2018)

61. G.W. Gibbons, M.J. Perry, C.N. Pope, Class. Quantum Gravity 22, 1503 (2005)

62. A. Ashtekar, S. Das, Class. Quantum Gravity 17, L17 (2000)

63. S. Das, R. Mann, JHEP 08, 033 (2000) 